Document downloaded from:

http://hdl.handle.net/10251/119576

This paper must be cited as:

El Fissi, L.; Fernández Díaz, R.; García Molla, P.; Calero-Alcarria, MDS.; García Narbón, JV.; Jiménez Jiménez, Y.; Arnau Vives, A... (2019). OSTEMER polymer as a rapid packaging of electronics and microfluidic system on PCB. Sensors and Actuators A Physical. 285:511-518. https://doi.org/10.1016/j.sna.2018.11.050

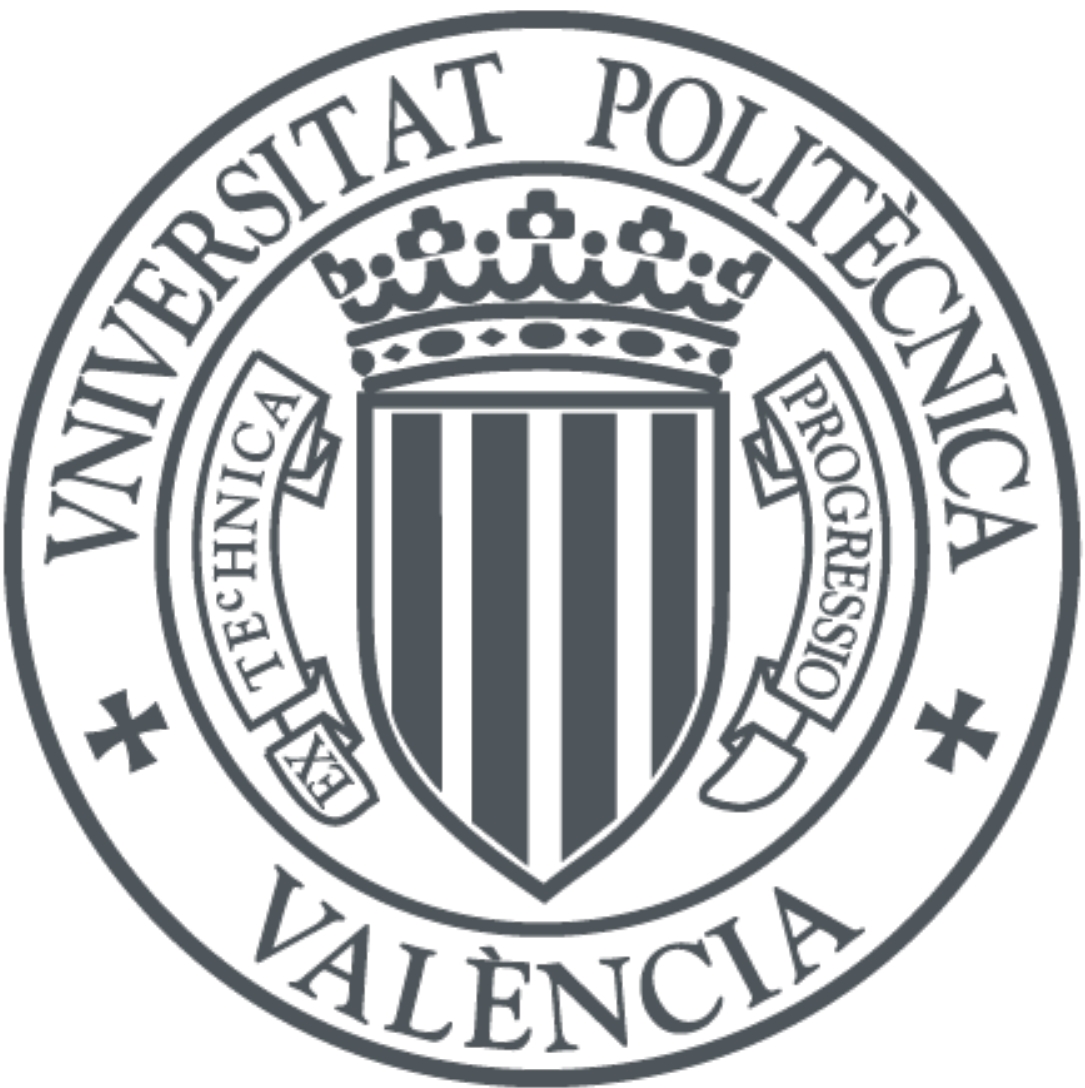

The final publication is available at

https://doi.org/10.1016/j.sna.2018.11.050

Copyright Elsevier

Additional Information 


\section{Accepted Manuscript}

Title: OSTEMER Polymer as a Rapid Packaging of Electronics and Microfluidic System on PCB

Authors: Lamia El Fissi, Román Fernández, Pablo García, María Calero, José V. García, Yolanda Jiménez, Antonio Arnau, Laurent A. Francis

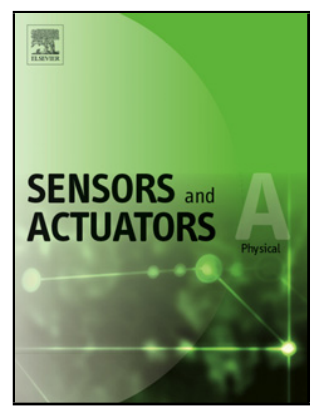

PII:

DOI:

Reference: S0924-4247(18)31706-0

To appear in: $\quad$ Sensors and Actuators A

Received date: $\quad 12$ October 2018

Revised date: $\quad 22$ November 2018

Accepted date: $\quad 27$ November 2018

Please cite this article as: El Fissi L, Fernández R, García P, Calero M, García JV, Jiménez Y, Arnau A, Francis LA, OSTEMER Polymer as a Rapid Packaging of Electronics and Microfluidic System on PCB, Sensors and amp; Actuators: A. Physical (2018), https://doi.org/10.1016/j.sna.2018.11.050

This is a PDF file of an unedited manuscript that has been accepted for publication. As a service to our customers we are providing this early version of the manuscript. The manuscript will undergo copyediting, typesetting, and review of the resulting proof before it is published in its final form. Please note that during the production process errors may be discovered which could affect the content, and all legal disclaimers that apply to the journal pertain. 


\title{
OSTEMER Polymer as a Rapid Packaging of Electronics and Microfluidic System on PCB
}

\author{
Lamia El Fissi*a, Román Fernández ${ }^{b}$, Pablo García ${ }^{b}$, María Calero ${ }^{b}$, José V. García ${ }^{b}$, \\ Yolanda Jiménez ${ }^{\mathrm{c}}$, Antonio Arnau ${ }^{\mathrm{c}}$, and Laurent A. Francis ${ }^{a}$
}

\author{
a* ICTEAM Institute, Université catholique de Louvain, Louvain-la-Neuve, Belgium. \\ ${ }^{\mathrm{b}}$ Advanced Wave Sensors S. L., Algepser 24, 46988 Paterna, Spain. \\ c Centro de Investigación e Innovación en Bioingeniería, Universitat Politécnica de Valéncia, \\ Camí de Vera S/N, 46022 Valencia, Spain. \\ *Corresponding author Tel:+(32)010472174 \\ Email address: lamia.elfissi@ uclouvain.be
}

Highlights

- A new heterogeneous integration method allows the integration of a microfluidic platform and a multichannel quartz crystal (150 MHz ) microbalance array on a printed circuit board (PCB).

- $\quad$ Dry adhesive bonding method is used.

- The microfluidic platform is replica molded using a UV-curable OSTEMER 322 Crystal Clear polymer.

- The resulting bond interface is shown to be completely homogeneous and void free.

- The package quality is tested to a differential pressure of up to 4 bars.

- The leak test of the cartridge is tested by pressurizing a microfluidic channel with an aqueous solution using an external peristaltic pump for more than 4 hours.

- $\quad$ The cartridge performance is evaluated by the electrical characterization.

\begin{abstract}
A new heterogeneous integration method is presented that allows the integration of a microfluidic platform and a multi-channel quartz crystal microbalance array on a printed circuit board (PCB) using a dry adhesive bonding method. In this work, the microfluidic platform is a replica molded using a UV-curable OSTEMER 322 Crystal Clear polymer. The OSTEMER acts both as a final package for the cartridge and as a functional material for hosting molded microfluidic channels, the input reservoirs and the waste reservoir. The method is demonstrated by the integration of an array of 24 of a $150 \mathrm{MHz}$ high fundamental frequency quartz crystal microbalance (HFF-QCM) to the OSTEMER microfluidic packaging. The resulting bond interface is shown to be completely homogeneous and void free, and the package is tested to a differential pressure of up to 4 bars. The leak test of the cartridge is performed by pressurizing a microfluidic channel with an aqueous solution using an external peristaltic pump for more than 4 hours. The cartridge performance is evaluated by the electrical characterization. Qfactor values of 9507 and of 650 are achieved in air and DI water, respectively. Results show that this simple integration method of the HFF-QCM is a promising way to integrate microfluidics into the more complex heterogeneous system.
\end{abstract}

Keywords: Microfluidic platform; Printed Circuit Board; Adhesive bonding; OSTEMER Crystal Clear polymer; HFF-QCM. 


\section{Introduction}

The microfluidics field has the potential to enable new capabilities and applications in the areas of biology, chemistry and medicine, through the manifestation of the concepts of "Lab on a Chip" and "Micro Total Analysis System"[1]. Owing to their attractive advantages such as a large scale fabrication and a compact size, the great majority of microfluidic devices is based on polymers. Polymers such as polymethylmethacrylate (PMMA) [2], Polydimethylsiloxane (PDMS) [3], polyesters (Ps) [4], polycarbonate (PC) [5], and cyclic olefin copolymer (COC) [6] have been investigated for the microfluidics fabrication by using various processing techniques including cast molding [3], hot embossing [7], injection molding [8] and laser ablation [9]. The printed circuit board (PCB) is the standard platform for building most of the electronic systems today. The PCB technology for manufacturing, developed and driven by the consumer electronics industry for more than 60 years, has reached a high level of maturity and precision, enabling a large-scale integration of the electronic functions on a small package [10]. Therefore, microfluidic systems have also benefited from their integration in PCBs to produce highly functional and small sized integrated microfluidic devices [10-14]. However, it might be very complex and costly to integrate such components, as the manufacturing technologies and the materials used for each part are different, which makes the hybrid integration quite rare in microfluidics. In recent years, several researchers have reported different hybrid integration strategies to assemble both electronic and fluidic onto one common PCB $[15,16]$. One promising strategy is to fabricate microchannels directly on the PCB chip and then to integrate a driving part and sensors into the PCB chip to obtain small and portable microfluidic devices [17]. The second one is to bond the microsensor onto the PCB and then to add the microfluidic part [18]. For both strategies, a strong and tight cohesion is necessary between the integrated parts. Wu et al. [18] have presented a modular integration approach to combine microfluidics with standard electronic components onto a PCB by using an epoxy bonding technique. The epoxy has been dispensed and pulled into the cavity between the in targeted circuit and the PCB by capillary forces, leaving the centre area open for direct exposure of the chip to the fluid. A glass microfluidic part has then been bonded onto the assembly part using a PDMS adhesive. Li et al. [19] have fabricated a USB-driven microfluidic device in which the microelectrodes of the sensors and the microchannels have been integrated directly into the copper sheet on a PCB. To seal the copper microchannels, the fabricated PCB has been covered by a glass side using a UV-curable adhesive. Babikian et al. [20] have presented a packaging architecture for fluidic surface mount components in a microfluidic PCB using a dry film photoresist. The lamination technique has been used to make the different photoresist layers used for the linearized layer and for the microfluidic layer.

The materials and techniques used to assemble the different parts of the microfluidic system onto the PCB have their advantages in terms of cost, biocompatibility, and manufacturing procedures. However, each integration strategy is established for a specific electronic chip. In addition, the manufacturing procedures proposed previously consist of a high number of parts and require several fabrication steps. In addition, the fabrication techniques used could fragilise the electrical components during the integration steps, and could then lead to a non-functional final device. Furthermore, the core material of a PCB stack is Eglass reinforced epoxy laminate (FR4 for Flame Resistant-4), an intrinsically hydrophobic material [21], which requires a surface treatment before the assembly steps, in order to improve the cohesion for the effective sealing of the microfluidic part and the PCB electronic platform.

In this work, we present an easy method for a quick, reproducible, and robust assembly technique designed for the fabrication of a polymer microfluidic cartridge on the PCB. We report the use of the thermoset OSTEMER polymer that could be ready used to rapidly fabricate, in parallel, the linearized layer, which provides a flat, biocompatible surface for the microfluidic part, and to fabricate the microfluidic system. The OSTEMER parts have been manufactured by a replica molding technique once the desired mold was fabricated, and then bonded to the electronic interface onto the PCB.

The main strengths of the thermostat OSTEMER are the biocompatible, robust and direct covalent bonding of different heterogeneous materials without surface treatment [22]. The OSTEMER polymer exhibits many similarities with PDMS, such as rapid prototyping and uncomplicated processing, However, PDMS comes along with several limitations, including high compliance resulting in channel deformation, high water vapour permeability leading to bubble propagation, the incompatibility with organic solvents, high permeability for small hydrophilic molecules as well as the presence of uncured polymer oligomers affecting the cellular physiology.

In order to validate our assembly technique, a multichannel monolithic quartz crystal microbalance (MQCM) has been used. Firstly, the fabrication technique of the different OSTEMER microfluidic parts has been described. Secondly, a detailed description of the assembly procedure steps for the fabrication of a microfluidic cartridge on PCB has been presented. The bonding quality of the different OSTEMER parts has been evaluated by using a leak test, pressurizing the microfluidic channels with an aqueous solution using a peristaltic pump and a pressure test. The performance of the cartridge has been evaluated by the electrical characterization oh the microsensor array with and without deionized (DI) water.

\section{Materials and Fabrication}

\subsection{Conceptual approach}

Our approach focuses on the integration of the microfluidic system on the bonded HFF-QCM microsensor array onto the PCB. The acoustic wave microsensor array is composed of 24 microsensors per array. To provide a flat surface between the microfluidic system and the assembled "PCB + Microsensor array", a leveling layer is required. This layer is composed of six 
microchannels, which surround the multi-channel quartz crystal microbalance. The microfluidic part is composed of six channels per microsensor array in such a way that each channel will be composed of four microsensors that can be used for different analysis sample assays. All the channels have the same length, so that the length has no effect on the flow pressure. In addition, six input reservoirs and one waste reservoir with an outlet hole are added to the design of the microfluidic part. The waste reservoir is connected to a peristaltic pump (Fig. 1).

The integration approach involves three steps, as depicted in Fig. 2. Firstly, the microsensor array has been mounted on the PCB. After the OSTEMER fabrication of the leveling layer and the microfluidic part, both parts have been bonded together to obtain a closed microfluidic system. Finally the closed microfluidic system has directly been aligned and bonded over the assembled "PCB + Microsensor array".

\subsection{Materials}

The commercially available UV-thermally cured OSTEMER crystal clear 322 [23] (Mercene Labs, Sweden) is used to manufacture the leveling layer and the microfluidic part. This polymer is based on the off-stoichiometry thiol-ene-epoxies and features a two-step curing process. It is initially shaped by using the UV-casting and it is then hardened and bonded to itself or other materials using a second thermal cure [24]. The OSTEMER can be molded by the casting technique using microstructured molds, such as photolithographically defined SU-8 / or KMPR structures on silicon wafers with Teflon coating, and a polydimethylsiloxane (PDMS) mold [22]. The injection molding technique can also be used for a quick and mass manufacturing [25]. In our case, the casting technique is chosen to manufacture the microfluidic part by using a PDMS mold (Sylgard 184, Dow Corning). An acoustic wave inverted mesa resonator microsensor array with a fundamental resonance frequency of 150 MHz MQCM (Advanced Wave Sensors S. L., Paterna, Valencia, Spain) have been used to validate our integrated approach. The microsensor array has been mounted over a custom PCB using a conductive epoxy (AADUCT 905, silver based). The PCB has been designed to provide mechanical stability to the resonator array and to act as a robust electrical interface between the sensors and the network analyzer. Bonding the QCM microsensor array on the PCB substrate improves robustness and handing. Since the wafer thickness is $66 \mu \mathrm{m}(10 \mu \mathrm{m}$ in the inverted-mesa regions), the array is very fragile and difficult to handle. Once it is bonded to the PCB, end -users can manipulate the array directly with their hands. Furthermore, the electrical interface connection between the array and the measurement system becomes simple, because it takes advantage of the PCB substrate to provide an easy and robust plug-and-play mechanism to the user based on a set of spring contacts.

The PCB has been made up of 2 conducting layers and using FR4 as substrate material. Reference marks have been included in the PCB legend for the alignment of the microfluidic setup. The PCB is rectangular, with dimensions $36 \mathrm{~mm} * 52 \mathrm{~mm} *$ $2 \mathrm{~mm}$. In Fig. 3, the resulting assembled" PCB + Microsensor array" is shown. The average of the fundamental frequency of the 24 microsensors onto the array in the air is $149.1 \mathrm{MHz}$.

\subsection{Fabrication process}

Before starting the fabrication process to manufacture the OSTEMER leveling layer and the microfluidic part, two Al molds have been fabricated using the micromilling technique. The first mold, for the OSTEMER leveling layer, is made of a $3 \mathrm{~mm}$ thick $\mathrm{Al}$ and composed of a six-microchannels groove (1 $\mathrm{mm}$ deep and $1 \mathrm{~mm}$ wide) and one circular groove for the outlet hole ( $2.5 \mathrm{~cm}$ diameter). The second mold, for the OSTEMER microfluidic part, is made of a $10 \mathrm{~mm}$ thick $\mathrm{Al}$ and composed of a six input circular reservoirs groove $(3.7 \mathrm{~mm}$ diameter; $6 \mathrm{~mm}$ deep) and one waste rectangular reservoir groove $(6 \mathrm{~mm}$ wide, 15 $\mathrm{mm}$ long; $4 \mathrm{~mm}$ deep) and a six-microchannels groove ( $0.1 \mathrm{~mm}$ deep and $1 \mathrm{~mm}$ wide). Both $\mathrm{Al}$ molds have been used to manufacture two other PDMS molds, which will be used to replicate the OSTEMER leveling layer and the microfluidic part. Prior to the fabrication of the PDMS molds, the $\mathrm{Al}$ inserts have been cleaned and dried. The PDMS precursor and a curing agent have been mixed at a ratio of 10 to 1, based on weight, and degassed in a vacuum desiccator. After removal of the bubbles, the polymer mixture has been cast in the Al cavity molds. The last step was the thermal curing of the PDMS in an oven with the ambient gas at $65^{\circ} \mathrm{C}$ for 2 hours, followed by peeling off the PDMS from the Al molds. Fig. 4 shows the PDMS molds for both the layers and the $\mathrm{Al}$ cavity inserts for each one.

The fabrication steps of the OSTEMER leveling layer and the microfluidic part using the casting technique and the direct bonding onto the assembled "PCB + Microsensor array" have been performed according to the fabrication scheme illustrated in Fig. 5.

At room temperature, the OSTEMER 322 Crystal clear component A (hardener) and component B (base) have been mixed at a ratio of 1.09 to 1 , based on weight, and degassed in a vacuum desiccator for one hour, prior to pouring the mixture into the PDMS mold (Fig. 5-1). Afterwards, both PDMS molds with the OSTEMER have been exposed to $365 \mathrm{~nm}$ UV light at $260 \mathrm{~W}$ during $60 \mathrm{~s}$ to ensure a minimum thiol epoxy reaction due to the heat generated by an excessive UV irradiation (Fig. 5-2). After that, the OSTEMER microfluidic part has been easily peeled off from the PDMS mold, and then manually aligned to the leveling layer (Fig. 5-3). The intermediately polymerized closed OSTEMER microfluidic system has been aligned manually and brought in direct contact with the assembled "PCB + Microsensor array". The alignment accuracy is about $1 \mathrm{~mm}$.

After that, a slight pressure has been applied by hand onto the bonded layer to evacuate the air trapped between the OSTEMER layers and the PCB. After a full polymerization step in the oven at $75^{\circ} \mathrm{C}$ for 3 hours, a small microtube piece $(0.7 \mathrm{~mm}$ ID, 2.3 mm OD; TYGON silicone tubing) was fixed manually in the outlet hole also using OSTEMER as a glue.

The bonded cartridge is presented in Fig. 6. The result reveals that the leveling layer defines perfectly the microchannels on the microsensor array, and that the microfluidic part is well bonded onto the leveling layer. No air gaps were are observed between the leveling layer and the assembled "PCB + Microsensor array". 
The key step in our integration approach is not only the application of the OSTEMER leveling layer to have a flat surface between the PCB and the microfluidic system, but also the bonding step order of the different OSTEMER parts. The application of the OSTEMER leveling layer followed by the microfluidic part reveals a bonding problem between both layers, though an air gap appears between the leveling layer and the assembled "PCB + Microsensor array". The source of the problem is the gap between the microsensor array and the top side of the PCB surface. This gap is due to the microsensor array thickness, and to the conductive epoxy glue used during the bonding step of the microsensor onto the PCB. So that the use of the OSTEMER closed microfluidic part (leveling layer + microfluidic part) provides a flat surface over the assembled "PCB + Microsensor array", since the closed OSTEMER microfluidic system is bonded perfectly onto the leveling layer even if an air gap could be present between the microsensor array and the leveling layer.

\section{Characterization of the integrated cartridge}

For microfluidic systems, the bond interface could be considered as a lifetime limiting factor, which directly affects the reliability of the devices. Depending on the bonding parameters, an intensive analysis of the bond interface is required to ensure the functionality of these systems. In our case, the bonding quality of the OSTEMER leveling layer and the microfluidic part is evaluated by using a pressure, and a leak test.

The device performance of a fabricated cartridge, including a resonance frequency and a quality factor are evaluated by using a network analysis with and without DI water.

\subsection{Pressure test}

In order to evaluate the bonding quality of the assembled layers and to examine the maximum pressure that our system could withstand, a pressure test was performed. Firstly between the OSTEMER leveling layer and the assembled PCB + Microsensor array, and then between the OSTEMER leveling layer and the OSTEMER microfluidic part. To this end, the PCB samples (1.5 $\mathrm{cm} * 1.5 \mathrm{~cm})$, the quartz samples $(1.5 \mathrm{~cm} * 1.5 \mathrm{~cm})$, and the OSTEMER samples $(1.5 \mathrm{~cm} * 1.5 \mathrm{~cm})$ have been directly bonded on different OSTEMER samples $(4 \mathrm{~cm} * 4 \mathrm{~cm}$, sample with the hole in the centre: entrance of pressurized air). Prior to the bonding step, the PCB and the quartz substrates have been washed in an acetone bath for 5 min and rinsed with Di water, then dried at ambient temperature. A hole was drilled in the centre of the OSTEMER sample so that a high pressure could be applied to break the sample at the bonding surface. Fig. 7 illustrates the experimental setup for the pressure test.

The pressure has slowly been increased until gas leaked out, or the pressure limit (4 bar) of the setup was reached. To ensure the adequate bond performance, the pressure test has been carried out by using 10 bonded samples for each bonding parameter. The bonding parameters (temperature and time17) and the failure pressure of the bonding interface created between the OSTEMER and the OSTEMER or PCB or quartz bonded samples are shown in Table 1.

Independently of the bonding parameters used to bond the different samples, the results revealed a bond burst pressure in excess of four bars. With such a pressure value, our bonded system could be used as a microfluidic system for our application, because the typical pressure encountered in a microfluidic Lab-on-Chip is about 1-2 bar [26].

\subsection{Leakage test}

The assembly " PCB + Microsensor Array + leveling layer + microfluidic part" (as shown in Fig. 6) has been connected to a peristaltic pump (Miniplus 3 Gilsons) using the connector outlet tube $(2.5 \mathrm{~cm}$ diameter) as shown in Fig. In our case, the pump provides a positive pressure.

To demonstrate the function of the fabricated cartridge, a red dyed DI water has been sucked through the microchannels at varying flows (from $3 \mathrm{ml} / \mathrm{min}$ to $20 \mathrm{ml} / \mathrm{min}$ in $3 \mathrm{ml}$ step) and examined under an optical microscope (Nikon, Instruments Europe B.V). The results obtained are shown in Fig. 9. Even after 4 hours with a flow of $10 \mathrm{ml} / \mathrm{min}$, no solution leakages are observed, between the leveling layer (which surrounds the microsensor array) and the OSTEMER microfluidic part (which includes the reservoirs and the channels to the microsensor array), which proves that the red dye does not penetrate the interfaces. According to these results, the microfluidic part was successfully sealed onto the microsensor array despite the presence of bubbles trapped in the reservoir, an issue that could be resolved by modifying the design of the microfluidic channels.

\subsection{Electrical characterization of the cartridge}

A network analyzer DG8SAQ VNWA 3 (1 kHz to $1.3 \mathrm{GHz}$ (SDR-Kits, Melksham, Wiltshire, UK) has been used to characterize the assembled cartridge performance by measuring the real and the imaginary parts of the electrical admittance spectrum of each microsensor on the array. All the sensors integration in the array have their top electrodes grounded, i.e. the ones in contact with liquid. Since they are connected to a fixed potential, the sample solution conductivity does not produce any significant channel interference. The dielectric constant of the raw materials of our cartridge is summarized in Table 2 .

Fig. 10 shows an example of a real and imaginary part of one resonator on the array after the assembly approach. All microsensors present in the array have the same expected admittance characteristics as shown in Fig. 10.

In order to evaluate the quality of the OSTEMER microfluidic packaging, the quality factor (Q-factor) of each microsensor 
was measured before and after the assembly step in air by using the network analyzer. In addition, in order to investigate the mechanical and electrical behaviours of the microsensor array in liquid media, DI water has been used.

For the microsensor measured in Fig. 10, a Q-factor of 17609 had been measured in air of the assembled " PCB + Microsensor array" (without the OSTEMER pakaging), while a Q value of 975.12 was obtained from the same microsensor with the OSTEMER microfludic packaging. The Q-factor value fell to 105.6 in DI water solution.

In air, the Q-factor value decreased dramatically after the assembly steps. It seems that the HFF-QCM microsensor array suffered from a lot of mechanical stress due to the bonding of the OSTEMER microfluidic parts. Generally, the degradation is unavoidable when the QCM chip is packaged using the standard packaging methods that use the screw mounted clamping, which induce stress on the microsensor and dampen the oscillation [27, 28]. It was recently reported by Sandstrom et al [29] that the use of the OSTEMER polymer as packaging of the QCM sensor presented less damping in the QCM and a high Qfactor. The OSTEMER part covalently bonded, i.e. not featuring externally applied clamping forces on the QCM, does not induce a lot of stress on the crystal and does not dampen the oscillation [24]. However, according to our results, something happened during the bonding steps. As we can see in Fig. 10, the fundamental frequency of the microsensor that is measured is slightly shifted (from $150 \mathrm{MHz}$ to $148.3 \mathrm{MHz}$ ) where can be explained by the fact that the resonance is disturbed by the addition of a small mass deposited on the surface of the acoustic resonator.

Fig. 11 shows a Field Emission Scanning Electron Microscope (FESEM) (ULTRA 55 ZEISS, Germany) characterizations of one of the microsensor (gold electrode) on the array before and after the bonding steps.

The FESEM recompositional analysis is summarized in Table 3.

According to the results, one can see that the gold electrode has been contaminated during the bonding steps (Fig. 11). Error! Reference source not found. reveals carbon as well as oxygen contamination adsorbed on the electrode surface. As the bonding steps have been provided in the clean room, and as no surface preparation has been used before the bonding steps, the results suggest that the contamination comes from the OSTEMER microfluidic part. It seems that during the bonding of the OSTEMER microfluidic part on top of the assembled " PCB + Microsensor array", a small and thin OSTEMER wire (appeared when the OSTEMER part had been peeled from the PDMS mold) has been adsorbed on the gold surface of the microsensors (Fig. 11-middle). After a full polymerization of the OSTEMER polymer, an OSTEMER agglomeration was formed on the gold electrode (Fig. 11-bottom).

The contaminated assembly "PCB + Microsensor array" with the OSTEMER leveling layer has been cleaned by using UV Ozone Cleaner (BioForce Nanosciences Inc., Chicago, IL, USA) for 10 min, rinsed with 99 (\%) ethanol, rinsed with DI water, dried with Nitrogen gas, and then treated for a second time with UV/ozone for $10 \mathrm{~min}$. It is worth noting that the OSTEMER is not affected by the use of the UV Ozone cleaner (optical microscope observation).

A high Q-factor has been recorded using the network analyzer, which is 11 times higher than the Q value before the cleaning step. For the microsensor measured in Fig. 10, the Q-factor is 9510 instead of 975.12 before the cleaning. The average Q-factor value of the 24 microsensors in the array after cleaning in the air is 9507.21. In DI water, a Q-factor value of 650 was measured after the cleaning instead of 105.56 before the cleaning.

As one can observe, the Q-factor value in DI water is reduced only by a factor of 1.5 (650 in water instead of 9510 in air). This fact can be explained if the penetration depth of the acoustic wave in the liquid medium is considered. As the resonance frequency becomes higher (150 MHz in our case), the penetration depth decreases together with the energy losses due to the liquid [30, 31].

\section{Conclusions}

We have successfully demonstrated a heterogeneous and easy integration approach, with a minimal number of parts and assembly steps, for the fabrication and the integration of the OSTEMER cartridge part onto the PCBs. A high fundamental frequency microsensors array has been used to validate our integrated approach. The resulting cartridges presented a good liquid sealing and a high Q-factor value. Q-factor values of 9510 and 650 have been measured by using a network analyser in air and water respectively. The results shew that the integrated HFF-QCM microsensors arrays are suitable for chemical, and biosensors applications in liquids. Furthermore, our integration approach is a promising way to integrate microfluidics into the more complex heterogeneous system.

\section{Acknowledgements}

This work was funded by the European Commission Horizon 2020 Programme under the Grant Agreement number ICT-282015/687785-LIQBIOPSENS (Reliable Liquid Biopsy technology for early detection of colorectal cancer).

\section{References}

[1] C. D. Chin, V. Linder and S. Sia, Commercialization of microfluidic point-of-care diagnostic devices, Lab Chip, 2012, 12, 2118- 21134.

[2] A. Beaton, T. R. Cardwell, C.L. and, V. Sieben, F. Legiret, E. Waugh, P. Statham, M. Mowlem and H. Morgan, Lab-on- 
chip measurement of nitrate and nitrite for in situ analysis of natural waters, Environmental Science and Technology, 2012, 17, 9548-9556.

[3] J. C. McDonald, D. C. Duffy, J. R. Anderson, D. T. Chiu, H. Wu, O. J. A. Schueller and G. M. Whitesides, Fabrication of microfluidic systems in poly(dimethylsiloxane), Electrophoresis, 2000, 21, 27-40.

[4] E. Young, E. Berthier, D. Guckenberger, E. Sackmann, C. Lamers, I. Meyvantsson, A. Huttenocher and D. Beebe, Rapid prototyping of arrayed microfluidic systems in polystyrene for cell-based assays, Anal Chem, 2011, 4, 1408-1417.

[5] N. Kim, M. Murphy, S. Soper and D. Nikitopoulos, Liquid-liquid segmented flows in polycarbonate microchannels with cross-sectional expansions Journal of Multiphase Flow, 2014, 58, 83-96.

[6] L. EL Fissi, D. Vandormael and L. A. Francis, Surface functionalization of cyclic olefin copolymer (COC) with evaporated TiO2 thin film, Sensors and Actuators A: Physical, 2015, 223, 76-83.

[7] H. Shadpour, H. Musyimi, J. F. Chen and S. A. Soper, Physiochemical properties of various polymer substrates and their effects on microchip electrophoresis performance, Journal of Chromatography A, 2006, 1111, 238-251.

[8] F. Dang, O. Tabata, M. Kurokawa, A. Ewis, L. Zhang, Y. Yamaoka, S. Shinohara, Y. Shinohara, M. Ishikawa and Y. Baba, High-performance genetic analysis on microfabricated capillary array electrophoresis plastic chips fabricated by injection molding, Anal Chem, 2005, 15, 1878-10.

[9 ] C. K. Chung, Y. C. Lin and G. R. Huang, , Direct writing on glass using a low-power UV laser in the manufacture of a microfluidic chip Journal of Micromechanics and Microengineering, 2005, 7, 2140-6.

[10] L. W. Liang, B. Sarkis, L. Guann-Pyng and B. Mark, Microfluidic printed circuit boards, Electronic Components and Technology Conference (IEEE), 2011.

[11] Y. Fu, Q. Yuan, J. Guo, Lab-on-PCB-based micro-cytometer for circulating tumor cells detection and enumeration, Microfluidics \& Nanofluidics, 2017, 21(2):20.

[12] C. Ren, S. Zhang, D. Song, J. Guo, Lab on dielectric film deposited PCB device for characterization of electrical property of biological cells, IEEE Transactions on Dielectrics \& Electrical Insulation, 2016, 23(4):1895-1897.

[13] J. Guo, H. Li, Y. Chen, Y. Kang, A Microfluidic Impedance Cytometer on Printed Circuit Board for Low Cost Diagnosis, IEEE Sensors Journal, 2014, 14(7):2112-2117.

[14]Moschou et al, Lab Chip, 2017,17, 1388-1405

[15] J. Guo, C. M. Li, Y. Kang, PDMS-film coated on PCB for AC impedance sensing of biological cells, Biomedical Microdevices, 2014, 16(5):681-686.

[16] D. Shi, J.GUO, L. Chen, C. Xia, Z. Yu, Y. Ai, C. M. Li, Y. Kang, Z. Wang, Differential microfluidic sensor on printed circuit board for biological cells analysis[J]. Electrophoresis, 2015, 36(16):1854-1858.

[17] A.Wego, S. Richter and L. Pagel, Fluidic microsystems based on printed circuit board technology, Journal of Micromechanics and Microengineering, 2001, 11, 528.

[18] A. Wu, L. Wang, E. Jensen, R. Mathies and B. Boser, Modular integration of electronics and microfluidic systems using flexible printed circuit boards, Lab on a Chip, 2010, 4, 519-21.

[19] J. Li, Y. Wang, E. Dong and H. Chen, USB-driven microfluidic chips on printed circuit boards, Lab on a Chip, 2014, 5, 860-4.

[20] S. Babikian, G. P. Li and M. Bachman, Packaging Architecture for Fluidic Components in Microfluidic PCBs, Electronic Components and Technology Conference (ECTC), 2016 IEEE 66th, 2016, 244, 528.

[21] N. Vasilakis, D. Moschou, D. Carta, H. Morgan and T. Prodromakis, Long-lasting FR-4 surface hydrophilisation towards commercial PCB passive microfluidics, Applied Surface Science, 2016, 368, 69-75.

[22] F. Saharil, L. El Fissi, Y. Liu, F. Calborg, D. Vandormael, L. A. Francis, W. Wijngaart and T. Haraldsson, Superior dry bonding of off-stoichiometry thiol-ene epoxy $(\operatorname{OSTE}(+))$ polymers for heterogeneous material labs-on-chip Proceedings of the 16th International Conference on Miniaturized Systems for Chemistry and Life Sciences, 2012, 1831-1833.

[23] http://www.ostemers.com/products.

[24] T. Haraldsson, C. F. Carlborg and W. Van der Wijngaart, OSTE - A novel polymer system developed for Lab-on-Chip, SPIE - International Society for Optical Engineering, 2014, 8976, 608-897.

[25] N. N Sandstrm, Z. Z. Shafagh, A. Vastesson, C. F. Carlborg, W. van der Wijngaart and T. Haraldsson, , Reaction injection molding and direct covalent bonding of OSTE+ polymr microfluidic devises Journal of Micromechanics and Microengineering, $2015,25,7$.

[26] S. Farizah, F. Carl, G. Tommy and W. van derWijngaart, Biocompatible "click" wafer bonding for microfluidic devices Lab Chip, 2012, 17, 3032-3037.

[27] L. Jinxing, D. Sheng, H. Jia and U, Toshitsugu, Flow-Injection-Based Miniaturized Quartz Crystal Microbalance Sensors and Materials, 2013, 7, 519-526.

[28] L. Jinxing, H. Jia, Z. Tian, L. Jing, Z. Xuefeng and U. Toshitsugu, An Experimental Study on Fabricating an Inverted Mesa-Type Quartz Crystal Resonator Using a Cheap Wet Etching Process Sensors, 2013, 9, 12140-12148.

[29] N. Sandstrom, R. Shafagh, B. Gylfason andW. Haraldsson, T. van derWijngaart, One step integration of gold coated sensors with OSTE polymer cartridges by low temperature dry bonding," in "16th International Conference on Solid-State Sensors, Actuators and Microsystems, 2011, pp. 2778-2781..

[30] Y. Montagut, J. Garca, Y. Jimnez, A. March, C. Montoya and A. Arnau, Validation of a Phase-Mass Characterization Concept and Interface for Acoustic Biosensors, Review of Scientific Instruments, 2011, 82, 064702. 
[31] R. Fernndez, P. Garca, M. Garca, Y. Garca Jos, V. Jimnez and A. Arnau, Design and Validation of a 150 MHz HFFQCM Sensor for Bio-Sensing Applications, Sensors, 2017, 17, 2057

\section{Biography}

Lamia EL FISSI obtained her PhD from University of Franche-Comte in Besancon (France) in 2010. Currently she did her postdoctoral researcher in Catholic University of Louvain UCL, Belgium. Her interests include design, fabrication and development of SAW sensors and the microfluidic devices for applications of lab-on-chip devoted to biology.

Laurent A. Francis (M.Eng. 2001, Ph.D 2006) is Associate Professor at the Electrical Engineering Department of the Université catholique de Louvain, Belgium. His main focus is on co-integrated, ultra-low power CMOS MEMS sensors for biomedical applications and harsh environments. He was previously researcher at IMEC in Leuven, Belgium, in the field of acoustic and optical biosensors and piezoelectric RF-MEMS. In 2011, he was visiting professor at the Université de Sherbrooke, Canada. He has published about 70 research papers in international journals, he is co-editor of one book, and holds one patent. He is a regular member of the IEEE.

\section{Figures}

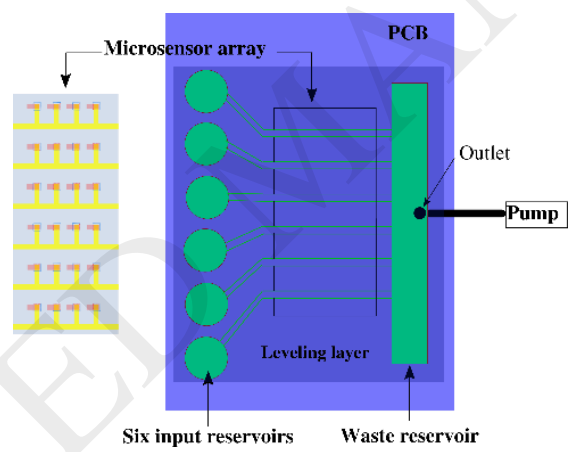

Fig. 1: Top view of the microfluidic cartridge on the PCB

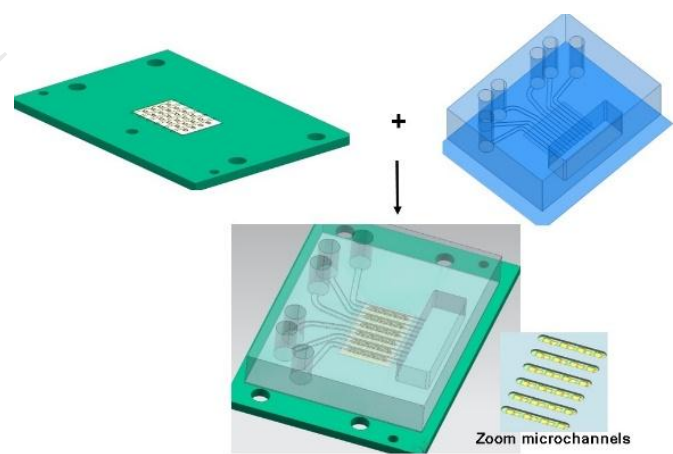

Fig. 2: 3D schematics of the integrated OSTEMER layers on the assembled"PCB + Microsensor array". 


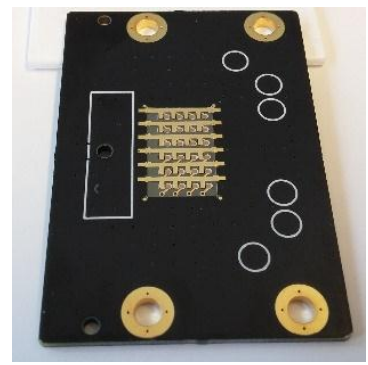

Fig. 3: Image of the HFF-QCM microsensor array mounted over the PCB using a conductive epoxy.
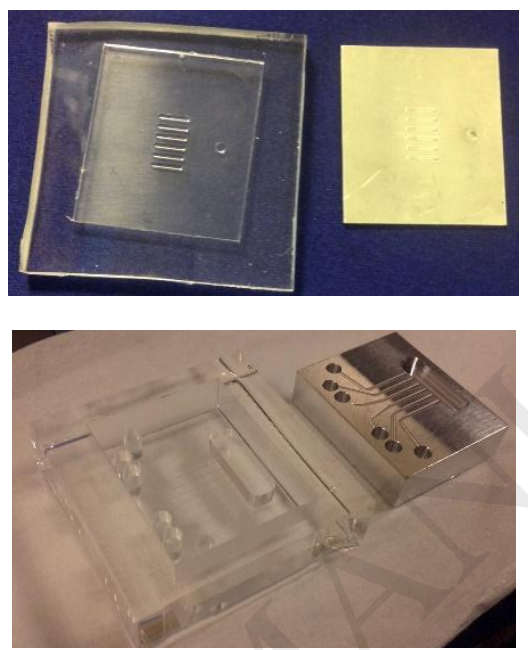

Fig. 4: Photography images of PDMS molds peeling off from the $\mathrm{Al}$ mold inserts fabricated using the micromachining technique. Top image: Al mold and PDMS mold used to manufacture the leveling layer; Bottom image: Al mold and PDMS mold used to make the microfluidic part.

OSTEMER

1. Casting

8

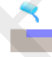

PDYS

2. Photocuring

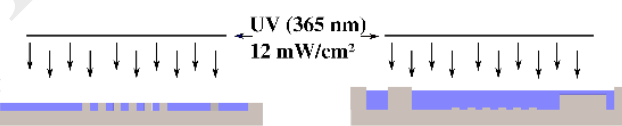

3. Demolding \& assembly

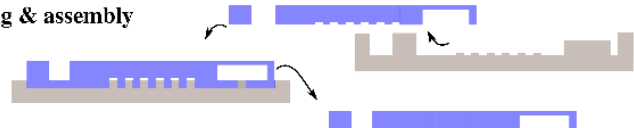

4. Thermal curing $\&$ bonding

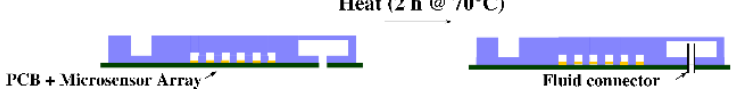

Fig. 5: Schematic illustration of the process to manufacture the OSTEMER leveling layer and the microfluidic part (1 \& 2), and to make the complete cartridge trough the bonding of the fluidic assembly (3) into the assembled "PCB + Microsensor array" (4). 


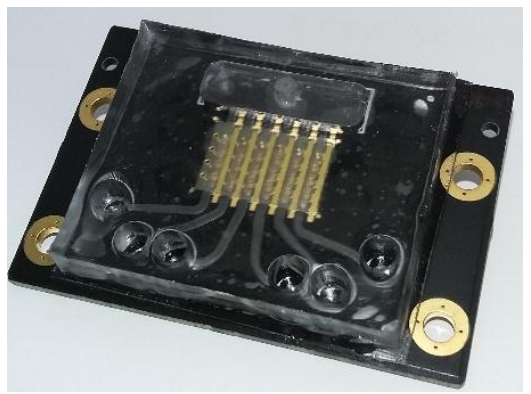

Fig. 6: Assembled system including the closed OSTEMER microfluidic system and the assembled"PCB + Microsensor array".

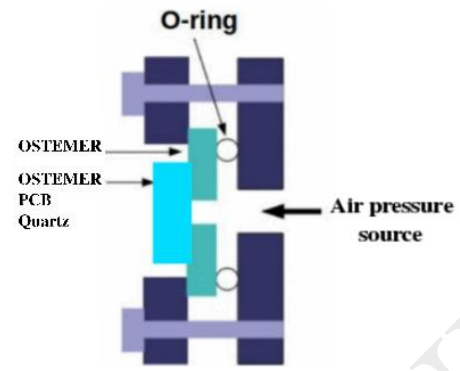

Fig. 7: The experimental setup of the pressure test.

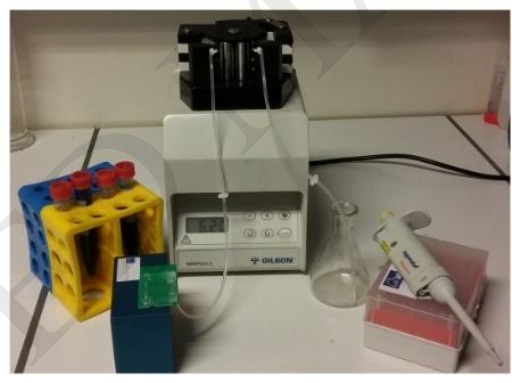

Fig. 8: The experimental setup of the leakage test.

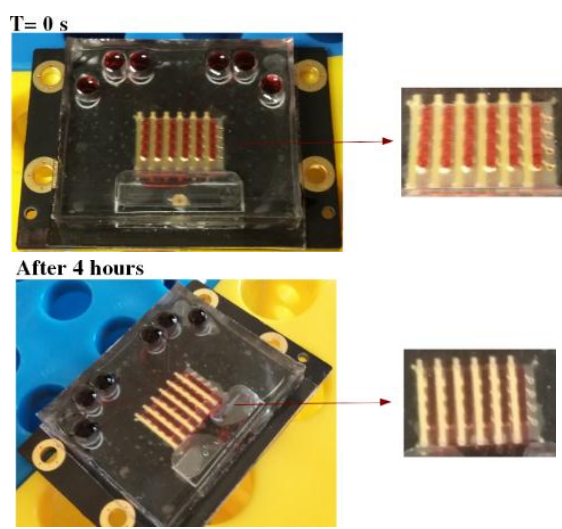




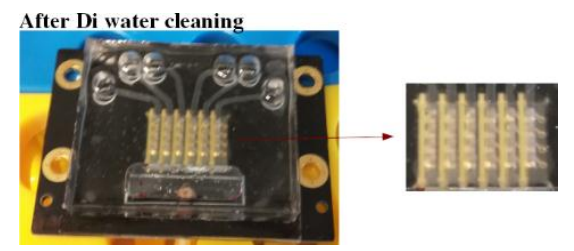

Fig. 9: Leakage test results.
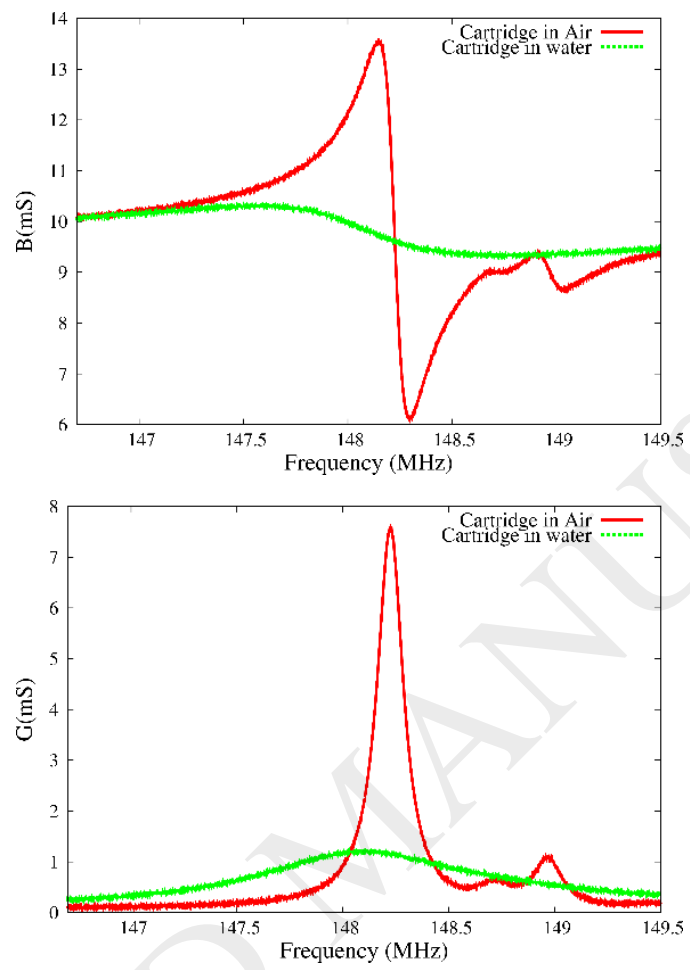

Fig. 10: Admittance Spectrum ((real (G) and imaginary (B) parts) of one microsensor in the assembled cartridge in air and DI water.

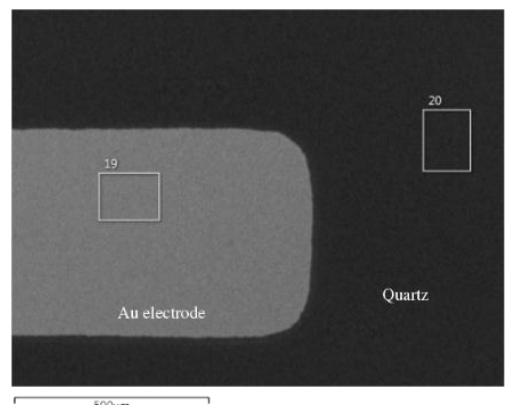



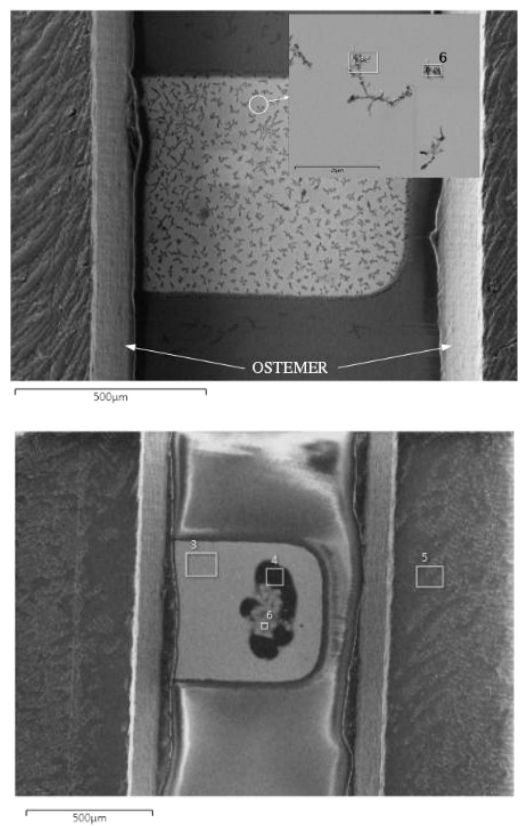

Fig. 11: FESEM Characterization of one microsensor in the cartridge. Top image: microsensor without OSTEMER; Middle image: microsensor with OSTEMER part (UV + RT 40h); Bottom image: microsensor with OSTEMER part (UV $+1 \mathrm{~h}$ at $\left.110{ }^{\circ} \mathrm{C}\right)$.

Table 1: Parameters pressure test and the results.

\begin{tabular}{|c|c|c|c|c|}
\hline $\mathrm{T}\left({ }^{\circ} \mathrm{C}\right)$ & Time $(\mathrm{h})$ & \multicolumn{3}{|c|}{ Pressure measured (bar) } \\
\hline R.T & 40 & & & \\
\cline { 1 - 2 } $36-48$ & 24 & Ostemer/PCB & Ostemer/Quartz & Ostemer/Ostemer \\
\cline { 1 - 2 } 70 & 3 & 4 & 4 & 4 \\
\hline 110 & 1 & & & \\
\hline
\end{tabular}

Table 2: Dielectric parameters of the cartridge components.

\begin{tabular}{|c|c|c|c|}
\cline { 2 - 4 } \multicolumn{1}{c|}{} & PCB & Quartz & OSTEMER \\
\hline Dielectric constant & $4.1^{1}$ & $3.78^{2}$ & $2.8^{3}$ \\
\hline
\end{tabular}

1: https://speedingedge.com/PDF-Files/tutorial.pdf

2: https://hypertextbook.com/facts/2008/JeffreyWong.shtml 3: Polymer data handbook

Table 3: FESEM compositional analysis of microsensor with and without OSTEMER fluidic part

\begin{tabular}{|c|c|c|c|c|}
\hline \multirow{2}{*}{ Image 11 } & \multicolumn{4}{|c|}{ Element concentration (\%) } \\
\cline { 2 - 5 } & $\mathrm{Au}$ & $\mathrm{C}$ & $\mathrm{O}$ & $\mathrm{Si}$ \\
\hline Top: area 19 & 97.8 & 0.72 & 0.64 & 0.86 \\
\hline Middle: area 6 & 85.42 & 8 & 5.1 & 1.4 \\
\hline Bottom: area 4 & 0 & 16 & 21 & 18 \\
\hline
\end{tabular}

